diastolic hypertension may be expected to be one-third to a half of those with a casual elevation of pressure ${ }^{12}$-that is, less than two million.

We cannot condone the indiscriminate labelling of persons as hypertensive when they have only a transient anxiety-related rise in blood pressure. These patients, if treated, may experience a marked reduction in blood pressure and such treatment may not be in the best interests of the patients. As Anderson has shown, ${ }^{3}$ from the Framingham data cardiovascular events do not increase until the diastolic blood pressure exceeds $90-99 \mathrm{~mm} \mathrm{Hg}$. There may be no benefit from reducing lower levels of diastolic pressure.

C J Bulpitt Diana Penfold

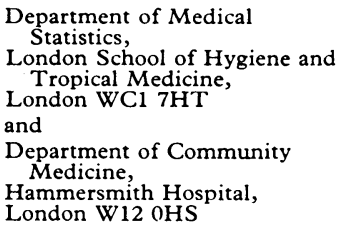

'Hawthorne VM, Greaves DA, Beevers DG. Br Med $\mathcal{f}$ 1974; iii:600-3.

2 Finnerty FA, Shaw LW, Himmelstach MD.

${ }^{3}$ Anderson TW. Lancet 1978; ii:1139.

\section{The coronary care controversy}

SIR,-We are grateful to Drs J M Rawles and A C F Kenmure (20 September, p 783) for drawing attention to two aspects of our randomised home-versus-hospital study in suspected myocardial infarction which merit fuller description.

The first is deaths before the arrival of the team. In our trial we dispatched a vehicle, with resuscitation equipment, a doctor, and a nurse, to patients who were registered with participating general practitioners and in whom a myocardial infarctio was suspected. Inevitably, calls for help were also received from other sources and for condition other than infarction and our paper reported on the first 500 calls which we had received, irrespective of their source. Of the 14 deaths before the arrival of the team, nine related to calls for help which had not come from participating doctors but from ambulance control, who asked us to dispatch equipment and a doctor to some special emergency (two children found unconscious at the bottom of swimming pool; a miner who collapsed at the coal face; four patients found collapsed by neigh bours or relatives; two patients who collapsed while non-participating GPs were with them and in whom external cardiac massage was already being performed when the team arrived but who remained in asystole). The remaining five death occurred in patients referred to us by participating doctors and are therefore the ones which directly relate to our study:

(1) Time between onset of symptoms and call to GP (patient delay) 90 minutes; from this call to GP's call for team (GP delay) 15 minutes; from call to team to their arrival (team delay) 35 minutes. Patient dead when team arrived.

(2) Patient delay 28 hours; GP delay 110 minutes team delay 20 minutes. Patient thought to have been dead for some time when team arrived. (3) Randomised to home group on day before death. GP paid routine visit but was recalled five hours later because of sudden collapse and asked team to visit. Patient dead when team arrived 17 minutes later. Necropsy showed acute haemorrhagic pancreatitis and no myocardial infarction. (4) Patient presented with one-week history of vague chest pain and was sent home from GP's surgery. GP asked team to go to house but on their arrival 30 minutes later the patient was dead.

5) Patient delay 110 minutes; GP delay 15 minutes; team delay 23 minutes. On arrival GP was performing external cardiac massage but heart could not be restarted.

Thus nine of the deaths reflect the pattern of emergencies in our area during the study period and five, which occurred in participating patients with suspected infarction, illustrate the problem of delay caused by patients and we are completing a study of ways of reducing this.

The second aspect we should like to amplify is our at-risk population. Drs Rawle and Kenmure assume that all our general practices began referring patients to us from the outset of the fouryear study period. Recruitment of each participating practice, however, required many briefing visits; so recruitment was serial and only the two largest practices were involved in the study for the whole four years. Their conclusion that we randomised "a mere $12 \%$ of the estimated number of coronaries" is thus invalid. Moreover, although it is necessary to remember this relationship between our randomised patients and the total infarct population if one wishes to apply our study to other communities, it does not invalidate the conclusion which we drew from the randomised patient themselves-namely, that for the majority of patients to whom a general practitioner had been called because of suspected infarction subsequen hospital admission conferred no advantage.

There are many considerations to take into account in planning coronary care for a community and our subsequent studies will, we hope furnish useful information on our ability to shorten patients' delays by an intensive advertising and educational campaign and on the value of restructuring the ambulance services to take accoun of the emerging Association of Emergency Medical Technicians. The results of these studies should add to a growing body of information which will enable the debate about community coronary care to be conducted within a rational rather than an emotional frame of reference.

J D HILI

J R HAMPTON

Department of Medicine,

J R A Mitchel

University $\mathrm{Hospi}$

Nottingham NG7 2UH

Heart attack, stroke, diabetes, and hypertension in West Indians, Asians, and whites

SIR,-The findings of $\mathrm{Dr} \mathrm{J} \mathrm{K}$ Cruickshank and others (25 October, $p$ 1108) that heart attack rates in West Indian immigrants are lower than expected is not surprising when one considers they have spent the earlier part of their life in a country where myocardia infarction and its risk factors are uncommon. The exceptional factor is hypertension, which when considered alone does not appear to be a risk factor in the aetiology of myocardia infarction in Jamaica. ${ }^{1}$

My own experience while working in a semirural community in Ife, Nigeria, would support this. In a one-year period (1977) the new cases of hypertension, stroke, diabetes mellitus, and heart attack seen by the medical department of one small hospital were: hypertension 84 , stroke 27 , diabetes 32 , myocardia infarction nil. As in the Jamaican study, the influence of affluence and Western diet, smoking, and sedentary occupation were low. Hypertension accounted for over $50 \%$ of cardiovascular disease, and was a considerable cause of mortality, usually from cerebral haemorrhage, hypertensive heart failure, or renal failure. Despite the high prevalence of diabetes, no case of myocardial infarction was seen over a three-year period.

In a Western environment people smoke more and eat more, especially in the form of "junk" foods, and they are certainly more sedentary. Thus in black immigrants we now see patients with myocardial infarction in the UK (albeit a lower incidence) and, of course, there are reports of myocardial infarction from the more affluent patients in the urban areas of countries such as Jamaica and Nigeria. Will there still be a difference in second-generation blacks born in this country ? If so, then we can start wondering more about the role of high blood pressure. To explain the difference between Asian and West Indian immigrants one needs to examine more closely the differences in other risk factors.

S J BeNTLEY

Withington Hospital,

${ }^{1}$ Ashcroft MT, Desai P. Lancet 1978;i:1167-70.

\section{Popliteal cyst rupture in normal knee joints}

SIR,-The paper by Drs D G Macfarlane and P A Bacon (1 November, p 1203) is a welcome addition to the literature on the painful swollen calf. In the last three months I have seen three ruptured Baker's cysts in the course of general medical admissions. Only one was associated with rheumatoid arthritis, the other two occurring in fit young men.

A 28-year-old civil servant had experienced intermittent swelling of the left knee joint over the two years following a cartilage extraction, the episodes being related to playing football. Occasionally a swelling at the back of the knee joint was present simultaneously. Over the week before admission such a swelling had been present, but it had disappeared on the day before admission in synchrony with the onset of paraesthesia in the left calf. Clinical examination showed there to be a red, tender, swollen calf and an effusion in the left knee joint. Pitting oedema was present over the shin and calf but there was no pedal oedema. Symptoms and signs settled rapidly with rest.

A 26-year-old scaffolder fell nearly 4 metres and landed on a straightened left leg two weeks before admission. The leg was painful but he continued to work normally. He was awoken by a tight discomfort in the left calf. On the following day he was found to have a red, tender, swollen left calf associated with a large knee effusion, a fullness in the popliteal fossa, and pitting oedema over the lower leg but not over the foot. Conservative treatment brought about a resolution of the inflammation within 24 hours.

In the management of a painful swollen calf a full history and careful examination of the whole of both legs are essential. ${ }^{1}$ It has been suggested that ruptured Baker's cysts are clinically indistinguishable from calf deepvein thromboses. ${ }^{2}$ In the two cases reported here definitive pitting oedema was present over the shin and calf but absent over the foot; in the case of the rheumatoid patient pitting oedema was disproportionately severe over the lower leg compared with the foot. Deepvein thrombosis of the calf is often associated with pedal oedema but rarely is pitting oedema seen over the shin but not the foot. I venture to suggest that this clinical sign may provide an additional aid to the diagnosis of ruptured Baker's cyst.

ROY TAYLOR

Newcastle General Hospital,

${ }^{1}$ Hughes GR, Pridie RB. Proc $R$ Soc Med 1970;63:
$587-90$.
2 Simpson FG, Robinson PJ, Bark M, Losowsky MS. Lancet 1980 ; i:331-3.

SIR,-The report by Drs D G MacFarlane and P A Bacon (1 November, p 1203) fails 